\title{
Blautia and Prevotella sequences distinguish human and animal fecal pollution in Brazil surface waters
}

\author{
Amber M. Koskey, ${ }^{1}$ Jenny C. Fisher, ${ }^{1}$ A. Murat Eren, ${ }^{2}$ \\ Rafael Ponce-Terashima, ${ }^{3}$ Mitermayer G. Reis, ${ }^{4}$ \\ Ronald E. Blanton ${ }^{3}$ and Sandra L. McLellan ${ }^{1 *}$ \\ ${ }^{1}$ School of Freshwater Sciences, University of Wisconsin \\ - Milwaukee, Milwaukee, WI 53204, USA. \\ ${ }^{2}$ The Josephine Bay Paul Center, Marine Biological \\ Laboratory, Woods Hole, MA 02543, USA. \\ ${ }^{3}$ Center for Global Health and Diseases, Case Western \\ Reserve University, Cleveland, $\mathrm{OH}$ 44106, USA. \\ ${ }^{4}$ Laboratory of Pathology and Molecular Biology, \\ Gonçalo Moniz Research Center, Salvador, Bahia 0000, \\ Brazil.
}

\section{Summary}

Untreated sewage discharges and limited agricultural manure management practices contribute to fecal pollution in rural Brazilian waterways. Most microbial source tracking studies have focused on Bacteroidales, and few have tested host-specific indicators in underdeveloped regions. Sequencing of sewage and human and animal feces with Illumina HiSeq revealed Prevotellaceae as the most abundant family in humans, with Lachnospiraceae and Ruminococcaceae also comprising a large proportion of the microbiome. These same families were also dominant in animals. Bacteroides, the genus containing the most commonly utilized human-specific marker in the United States was present in very low abundance. We used oligotyping to identify Prevotella and Blautia sequences that can distinguish human fecal contamination. Thirty-five of 61 Blautia oligotypes and 13 of 108 Prevotella oligotypes in humans were host-specific or highly abundant (i.e. host-preferred) compared to pig, dog, horse and cow sources. Certain human Prevotella and Blautia oligotypes increased more than an order of magnitude along a polluted river transect in rural Brazil, but traditional fecal indicator levels followed a steady or even decreasing trend. While both Prevotella and Blautia oligotypes distinguished human and animal fecal pollution in Brazil surface waters, Blautia appears to

Received 22 December, 2013; accepted 9 June, 2014. *For correspondence. E-mail mclellan@uwm.edu; Tel. 414382 1710; Fax 414 3821705. contain more discriminatory and globally applicable markers for tracking sources of fecal pollution.

\section{Introduction}

Fecal pollution in surface water sources constitutes a significant public health threat worldwide. Waterborne illnesses, related to the inadequate provision of water and sanitation services, are responsible for four billion cases of diarrhea and 1.8 million deaths each year, mostly impacting children five years of age and younger living in the rural communities of developing countries (Esrey et al., 2001; WHO, 2007). The lack of sanitation and drinking water infrastructures within these rural communities creates a self-perpetuating cycle of waterborne diseases (Esrey et al., 2001; WHO, 2013b). In Brazil, these range from diarrheal illnesses caused by a broad array of pathogens including Escherichia coli, Campylobacter, Giardia, Cryptosporidium, helminth and enteric viruses (Guerrant et al., 1983; Franzolin et al., 2005; Moreno et al., 2010) to schistosomiasis, a systemic parasitic disease with a morbidity/mortality rate estimated at over 250,000 per year (WHO, 2013a). While there has been significant progress in controlling schistosomiasis through repeated mass administration of drugs, it is still the second leading cause of death from parasitic infections in Brazil (Blanton et al., 2011).

Data from legally mandated fecal contamination surveillance in Brazil have shown that regions with the highest prevalence of diseases overlap with areas of low sanitation (Barreto et al., 2007). Although this relationship has been demonstrated, identifying human sources in waterways with fecal pollution is less certain. Untreated sewage discharges and limited agricultural manure management practices contribute to fecal pollution in Brazilian waterways (de Oliveira et al., 2012). Exposure to human feces is generally a greater health risk than exposure to animal feces (Soller et al., 2010); therefore, identifying the source contamination is important for risk assessment (USEPA, 2005; Field and Samadpour, 2007; Santo Domingo et al., 2007; Stewart et al., 2013). Traditional fecal indicator bacteria, such as E. coli and enterococci, are found in the feces of humans and animals alike, which limit their potential to accurately assess human health risk. Host-specific alternative indicators may therefore be 
particularly useful in areas where there is ubiquitous fecal pollution in surface waters (Newton et al., 2011; Roslev and Bukh, 2011).

The majority of research to identify host-specific genetic markers (i.e. a specific sequence within the DNA of fecal bacteria) have focused on Bacteroidales (Bernhard and Field, 2000; Dick et al., 2005; Reischer et al., 2006; Kildare et al., 2007; Fremaux et al., 2009). Geographic variability and host specificity can factor into the efficacy of markers in discrimination of host sources, (Bernhard and Field, 2000; Harwood et al., 2009; Shanks et al., 2013). Only a few studies have explored the applicability of these markers in underdeveloped regions (Jenkins et al., 2009; Ahmed et al., 2010; Reischer et al., 2013). One study in Kenya tested human and cow-specific Bacteroidales assays and demonstrated that the cowspecific assay could detect $94 \%$ of samples; however, detection of human fecal pollution was much less, with only $65 \%$ of human fecal samples positive with one human-specific assay (Jenkins et al., 2009). Reischer and colleagues (2013) found cattle and ruminant Bacteroidales markers were widely reliable in a study spanning across 16 countries on six continents; however, human Bacteroidales genetic markers were less prevalent and stable, suggesting more reliable markers were needed. Recent human microbiome studies demonstrate a difference in the microbial community across geography, age and time (De Filippo et al., 2010; Lozupone et al., 2012; Yatsunenko et al., 2012). Differences in cultures and diets may also cause variation in the human gut microbiota, which manifest in the relative abundances of Bacteroides versus Prevotella (De Filippo et al., 2010; Wu et al., 2011). Considering these findings, more research is needed to evaluate if 'human-specific' genetic markers developed in the United States and EU are relevant in other regions of the world (Reischer et al., 2013). Further, a broader array of taxonomic groups other than Bacteroidales may need to be explored to identify robust indicators that can be applied globally for tracking human sources of fecal pollution.

With deep sequencing technologies becoming more commonplace, it is now possible to identify multiple microorganisms that provide a fecal signature within a sample (Newton et al., 2013) more reliably than a single marker (Unno et al., 2010; Dubinsky et al., 2012; Newton et al., 2013). Given the complexity and depth of massively parallel, high-throughput sequencing data sets, the use of sensitive methods that can distinguish closely related but distinct organisms is critical for accurate identification of markers associated with certain hosts. Oligotyping is a recently described method that facilitates high-resolution partitioning of the 16S rRNA gene amplicon data into 'oligotypes' using Shannon entropy (Eren et al., 2013b). By recovering subtle nucleotide variation among reads, oligotyping can distinguish organisms with more than $99 \%$ identity over the sequenced region (Eren et al., 2011; 2013b). Previous studies identified oligotypes within the genus Blautia that distinguish human and animal sources with remarkable accuracy (Eren et al., 2014).

In this study, we used the Illumina HiSeq platform for ultra-deep sequencing of the bacterial communities associated with sewage, and fecal samples collected from humans and other animals including pig, dog, horse and cow. Processing and sequencing of sewage, and human and pig fecal samples is described elsewhere (Eren et al., 2014). Processing of dog, horse and cow fecal samples is described in Supporting Information Text S1, along with other methods specific to this study. We applied an extremely stringent quality filtering on our sequencing reads to eliminate the vast majority of sequencing errors (Eren et al., 2013a) to avoid false positives in our findings. We explored the oligotypes that can discriminate hosts, and evaluated their environmental applicability by analysing river water samples collected from the Jiquiriçá River in Jenipapo, Brazil and the Lucaia River in Salvador, Brazil. Ongoing work by our group examines the correlation of human fecal contamination in the Jiquiriçá River with risk of Schistosoma mansoni infection. We contribute to this goal by describing the oligotype signature profile in human samples from Brazil and the imprint of this signature on contaminated surface water.

\section{Results and discussion}

\section{Microbial community structure of human fecal and sewage samples in Brazil}

Brazilian human fecal samples and sewage shared common fecal taxa in similar proportions (Fig. 1), but sewage also contained taxa that have been previously associated with pipe infrastructure and surface water samples (McLellan et al., 2010; VandeWalle et al., 2012; Shanks et al., 2013). Prevotella, within the family Prevotellaceae, was the most abundant genus in the average Brazilian human fecal sample and also in Brazilian sewage. Genera belonging to the families Lachnospiraceae (e.g. Blautia, Roseburia) and Ruminococceae (e.g. Ruminococcus, Oscillibacter, Faecalibacterium) also made up a large proportion of the sewage and human fecal samples when combined (Fig. 1, Table 1). The latter two families have been identified as dominant families in US human fecal and sewage samples (Turnbaugh et al., 2009; McLellan et al., 2010; 2013). Notably abundant in US human fecal samples, but less prevalent in Brazil human fecal samples, are the Bacteroides, which make up $\sim 11 \%$ of US human fecal samples and $\sim 2 \%$ of US sewage (Dethlefsen et al., 2008; Turnbaugh et al., 2009; 


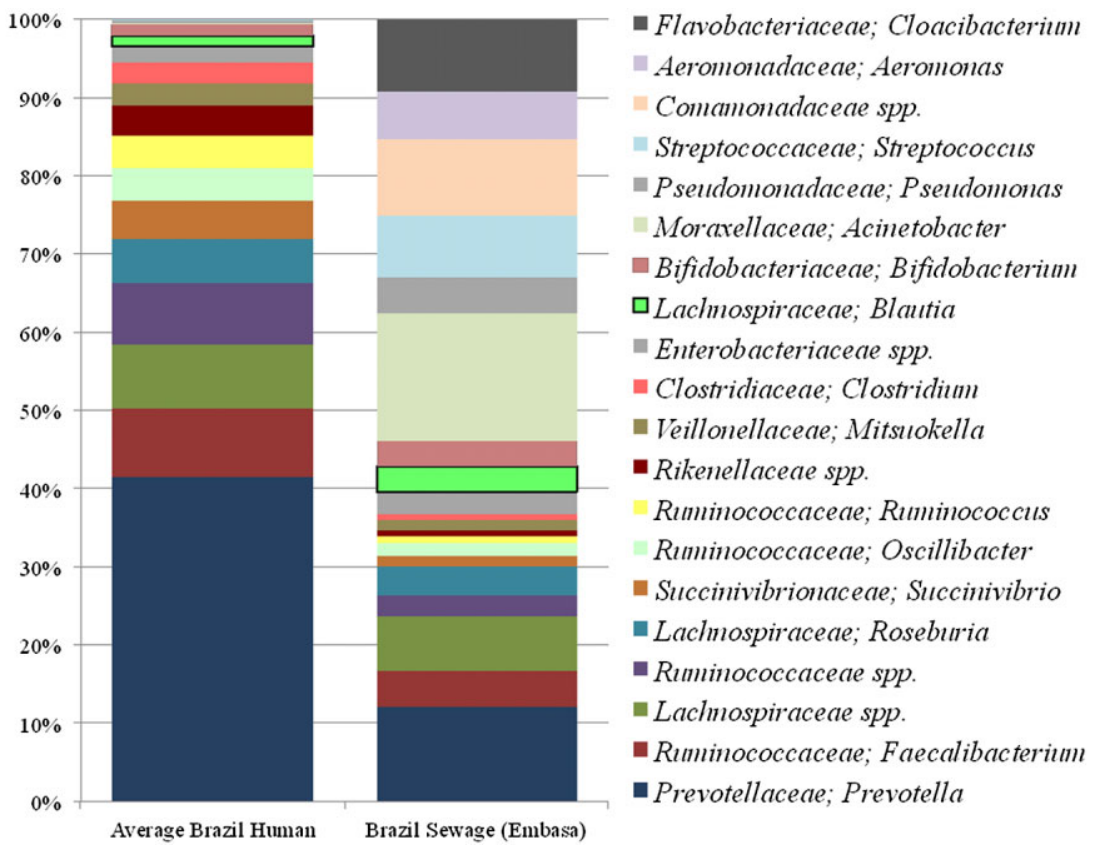

Fig. 1. Microbial community populations of sewage and human fecal samples collected in Brazil. A $50 \mathrm{ml}$ sewage sample was from Embasa (Empresa Baiana de Águas e Saneamento - The Bahian Water and Sanitation Company), a water treatment facility in Salvador, Brazil, on 13 August 2012. DNA extraction was carried out as described previously by our laboratory (McLellan et al., 2010). The human fecal samples $(n=10)$ were collected as part of a 2009 schistosomiasis survey (Blanton et al., 2011). The V6 hypervariable regions of the 16S rRNA gene from community genomic DNA were amplified and sequenced using Illumina HiSeq as described previously (Eren et al., 2013a). Taxonomy was assigned to sequences using Global Alignment for Sequence Taxonomy (GAST) (Huse et al., 2008), and taxonomic counts were normalized to the maximum number of sequences. Nearly 15 million bacterial sequence reads were generated ( 1 million reads per sample). Sequence data are available in the National Center for Biotechnology Information Short Read Archive under the accession number SRP041262. Taxonomic counts were normalized to maximum number of sequences, and abundance parameters were set from $1 \%$ to $100 \%$. The relative proportion of the top 20 genera occurring in sewage $(n=1)$ and humans (average of $n=10)$ are presented. The genera shown comprised $\sim 40 \%$ of the total sequence reads for both humans and sewage.

McLellan et al., 2010) but comprised $<1 \%$ of the Brazil human and sewage samples.

Populations consuming carbohydrate-rich diets in Africa and South America have higher proportions of Prevotella in their gut microbiomes, whereas Bacteroides tend to dominate the guts of individuals from Europe and North America who typically consume a higher protein diet (De Filippo et al., 2010; Wu et al., 2011; Yatsunenko et al., 2012). Our research further supports this geographical distinction, as the Brazilian human fecal and

Table 1. Average taxonomic composition of 14 fecal associated bacterial families in human fecal samples, sewage and 10 Brazilian animal fecal samples (three pigs, three dogs, two cows and two horses) collected from Jenipapo, Brazil, in August 2012. Data are based on the percentage of sequence reads associated with the taxonomic classification of these previously reported bacterial families (Turnbaugh et al., 2009; McLellan et al., 2010; Newton et al., 2013). These 14 families represented $26-50 \%$ of the total bacterial communities for each sample type.

\begin{tabular}{|c|c|c|c|c|c|c|}
\hline Family & $\begin{array}{l}\text { Embasa } \\
\text { Sewage \% }\end{array}$ & $\begin{array}{l}\text { Average } \\
\text { human \% }\end{array}$ & $\begin{array}{l}\text { Average } \\
\text { pig \% }\end{array}$ & $\begin{array}{l}\text { Average } \\
\text { dog } \%\end{array}$ & $\begin{array}{l}\text { Average } \\
\text { horse \% }\end{array}$ & $\begin{array}{l}\text { Average } \\
\text { cow \% }\end{array}$ \\
\hline Ruminococcaceae & 18 & 25 & 30 & 13 & 23 & 18 \\
\hline Prevotellaceae & 19 & 38 & 19 & 10 & 13 & 12 \\
\hline Lachnospiraceae & 27 & 20 & 16 & 10 & 18 & 8.3 \\
\hline Enterobacteriaceae & 5.4 & 2.0 & 1.4 & 36 & 11 & 20 \\
\hline Porphyromonadaceae & 4.8 & 0.50 & 7.3 & 8.4 & 5.8 & 10 \\
\hline Veillonellaceae & 7.9 & 4.4 & 11 & 3.2 & 3.8 & 2.7 \\
\hline Rikenellaceae & 1.5 & 3.6 & 4.6 & 3.2 & 12 & 7.0 \\
\hline Clostridiaceae & 1.3 & 2.3 & 1.7 & 3.5 & 4.1 & 8.1 \\
\hline Erysipelotrichaceae & 4.4 & 2.7 & 3.9 & 3.5 & 2.0 & 2.1 \\
\hline Bacteroidaceae & 4.9 & 0.30 & 0.30 & 3.8 & 3.1 & 3.4 \\
\hline Lactobacillaceae & 0.70 & 0.10 & 1.5 & 3.8 & 1.7 & 4.2 \\
\hline Bifidobacteriaceae & 4.9 & 1.4 & 0.30 & 0.90 & 1.0 & 0.60 \\
\hline Fusobacteriaceae & 0.30 & 0 & 1.9 & 1.8 & 0.90 & 3.7 \\
\hline Enterococcaceae & 0.10 & 0 & 0 & 0.10 & 0.10 & 0.10 \\
\hline
\end{tabular}


sewage samples had a higher abundance of Prevotella versus Bacteroides. In contrast to differences in the relative abundance of Bacteroides between the United States and Brazil, the genus Blautia had a consistent signal in both populations. On average, Blautia makes up $1.5 \%$ of US and $1.3 \%$ of Brazilian human fecal samples, $0.9 \%$ of US sewage and $2.0 \%$ of Brazilian sewage (McLellan et al., 2010; Newton et al., 2011; Eren et al., 2014).

The most widely used markers for human fecal contamination target human-specific Bacteroides spp., which have been found to be widely applicable to tracking sewage in many areas including the United States, Europe and Australia (Gourmelon et al., 2007; Reischer et al., 2007; Ahmed et al., 2009; 2010; Sauer et al., 2011). However, a lower sensitivity was reported in a study that included transcontinental comparisons, suggesting that there may be targets in the human microbiome better suited for microbial source tracking (Reischer et al., 2013). We recently described an assay, termed Lachno2, for a Blautia spp. found in humans but absent in cow and chicken samples and have used it to demonstrate chronic sewage contamination in a US estuary (Newton et al., 2011). Given the rich array of sequences within Blautia that can identify host sources, this taxonomic group shows promise for further alternative indicator development (Eren et al., 2014). Genetic markers for human fecal pollution based on Lachnospiraceae, and more specifically Blautia, may prove more universally applicable than Bacteroides, given the higher proportion of this taxonomic group within the Brazilian fecal and sewage samples. Furthermore, Prevotella may serve as a better target in regions such as rural Brazil, where the standard diet favours its dominance in the gut microbiome. Future studies could examine Prevotella population structure in humans across a broad geographic area to develop a human Prevotella targeted assay, either alone or in conjunction with a Blautia assay.

\section{Comparison of Brazil human and animal fecal microbial communities}

All animal samples contained 14 common fecal families, but in different relative proportions (Table 1); genera within these fecal families varied among samples as well. A comparison of the 14 most abundant fecal-associated families in Brazilian humans, animals and sewage showed that Ruminococcaceae had the highest relative abundance, making up $13 \%$ to $30 \%$ of each sample, followed by Prevotellaceae and Lachnospiraceae (Table 1). These three families comprised $>80 \%$ of the human sequence reads and $>60 \%$ of the sewage and pig samples, but were less abundant in the cow, horse and dog fecal samples. Enterobacteriaceae, a large family of facultative anaerobes that contain pathogenic members, made up $>11 \%$ of the dog, horse and cow fecal samples, $>5 \%$ of the sewage samples but $<2 \%$ of the human and pig fecal samples. Bacteroidaceae, the family containing the genus Bacteroides, comprised $~ 3 \%$ of the dog, horse and cow samples, but was considerably lower in the human and pig fecal samples. This particular suite of organisms, notably the top four fecal families, could be useful for tracking animal fecal contamination in the surface waters of Brazil. Studies have identified animalspecific markers based on Bacteroides; however, further study is needed to test their applicability to other regions of the world (Dick et al., 2005; Fogarty and Voytek, 2005; Fremaux et al., 2009; Mieszkin et al., 2010). These assays target the V2 to V4 regions, whereas our study targeted the $\mathrm{V} 6$ region. Therefore, additional full length sequencing and/or deep sequencing of additional regions is needed to make direct comparisons.

\section{Distribution of Blautia and Prevotella oligotypes among Brazil sewage, human and animal fecal microbial communities}

We utilized oligotyping to investigate sequences that could distinguish human fecal pollution from animal sources. We identified 61 Blautia oligotypes in the 10 human fecal samples, representing a total of 134595 sequence reads (Table 2; Fig. 2). Thirty-five oligotypes (79 469 sequence reads; 58\%) were human specific (found in all human samples and only human samples; $n=6$ ), human associated (found only in some human samples; $n=26$ ) or human preferred (found at significantly higher abundance in humans than other sources; $n=3$ ) when compared to the animals in our study (pigs, dogs, horses and cows). A total of 33 of the 35 human oligotypes were also present in the sewage sample from the Embasa wastewater treatment plant in Salvador, Brazil. The top three human-specific oligotypes comprised $11 \%$ of Blautia in humans and $6.2 \%$ of the sewage reads from the genus Blautia. Previous work in our laboratory has demonstrated that certain Blautia oligotypes are shared between US and Brazil sewage, suggesting some Blautia genetic markers may be universally applicable (Eren et al., 2014). Assays based on additional humanspecific Blautia oligotypes could be used in conjunction with the Lachno2 assay to enhance specificity of human source identification.

Although Prevotella was noted as the dominant organism in the Brazilian human fecal samples, the two most dominant Prevotella oligotype in humans were also dominant in animals (Table 2; Fig. 3). We did not find any human-specific oligotypes in Prevotella; however, nine of the 108 Prevotella oligotypes identified in the 10 human fecal samples were human associated, and another three 
Table 2. Relative abundance of human-related Blautia and Prevotella oligotypes.

\begin{tabular}{|c|c|c|c|}
\hline & $\begin{array}{l}\text { Sequence read } \\
\text { abundance }\end{array}$ & $\begin{array}{l}\% \text { of reads in genus } \\
\text { (from humans) }^{\mathrm{a}}\end{array}$ & $\begin{array}{l}\% \text { of reads in genus } \\
\text { (from sewage) })^{b}\end{array}$ \\
\hline \multicolumn{4}{|l|}{ Blautia } \\
\hline Total human oligotypes $(n=61)$ & 134,595 & $98 \%{ }^{c}$ & $97 \%{ }^{c}$ \\
\hline Human-specific, preferred or associated oligotypes $(n=35)$ & 79,469 & $58 \%$ & $32 \%$ \\
\hline \multicolumn{4}{|l|}{ Top three human-specific oligotypes } \\
\hline AACTCGGACCCCTAA & 9910 & $7.2 \%$ & $1.0 \%$ \\
\hline AACTCGTCTACTTGA & 3945 & $2.9 \%$ & $2.3 \%$ \\
\hline AGCTCACTCGTTTGA & 1151 & $0.8 \%$ & $2.9 \%$ \\
\hline \multicolumn{4}{|l|}{ Prevotella } \\
\hline Total human oligotypes $(n=108)$ & $3,792,564$ & $99 \%^{c}$ & $99 \%^{\mathrm{c}}$ \\
\hline Human-associated and preferred oligotypes $(n=12)$ & 114,255 & $3.0 \%$ & $1.6 \%$ \\
\hline \multicolumn{4}{|l|}{ Top three human-associated/preferred oligotypes } \\
\hline CCATACGTAGACTCTTCTG & 95,700 & $2.5 \%$ & $0.009 \%$ \\
\hline CCATACGATGACAATTCCG & 4,635 & $0.12 \%$ & $0.91 \%$ \\
\hline TCGGAAGATTGATACCGTG & 2,918 & $0.077 \%$ & $0.012 \%$ \\
\hline
\end{tabular}

a. Compared to total human Blautia or Prevotella sequence reads.

b. Compared to total sewage Blautia or Prevotella sequence reads.

c. A small percentage of total reads from human and sewage were not used in oligotyping after quality filtering.

were human preferred. Eleven of these oligotypes were represented in sewage. The top three human-preferred Prevotella oligotypes comprised $2.7 \%$ of all Prevotella in human samples and $1.0 \%$ of sewage samples.

Although Prevotella was the most abundant genus in terms of total sequence reads, the majority of the sequence reads were not specific to the mammals surveyed. The relative abundance of one of the humanpreferred Prevotella oligotypes was an order of magnitude higher than the human Blautia oligotypes, but Prevotella had no strictly human-specific oligotypes. In contrast, more than half of the Blautia oligotypes distinguished humans from animals. These results suggest that Blautia oligotypes may be a more discriminatory fecal source marker, while Prevotella oligotypes may offer sensitivity for detection. It should be noted that our sequence reads are very short and that higher diversity may occur in other regions of the Prevotella 16S rRNA gene (Fogarty and Voytek, 2005; Haugland et al., 2010) that could allow for discrimination of Prevotella from humans and animals. The combination of these two fecal indicators could be very useful for simultaneously tracking total fecal pollution as well as the relative contribution from humans.

\section{Tracking human fecal contamination in surface waters with oligotypes versus $\mathrm{E}$. coli and Enterococcus spp. sequence abundance}

The relative abundance of sequence reads identified as Prevotella, Blautia, E. coli and Enterococcus varied at sites along the Jiquiriçá River (Fig. 4). Prevotella spp. and Blautia spp. both increased considerably at site

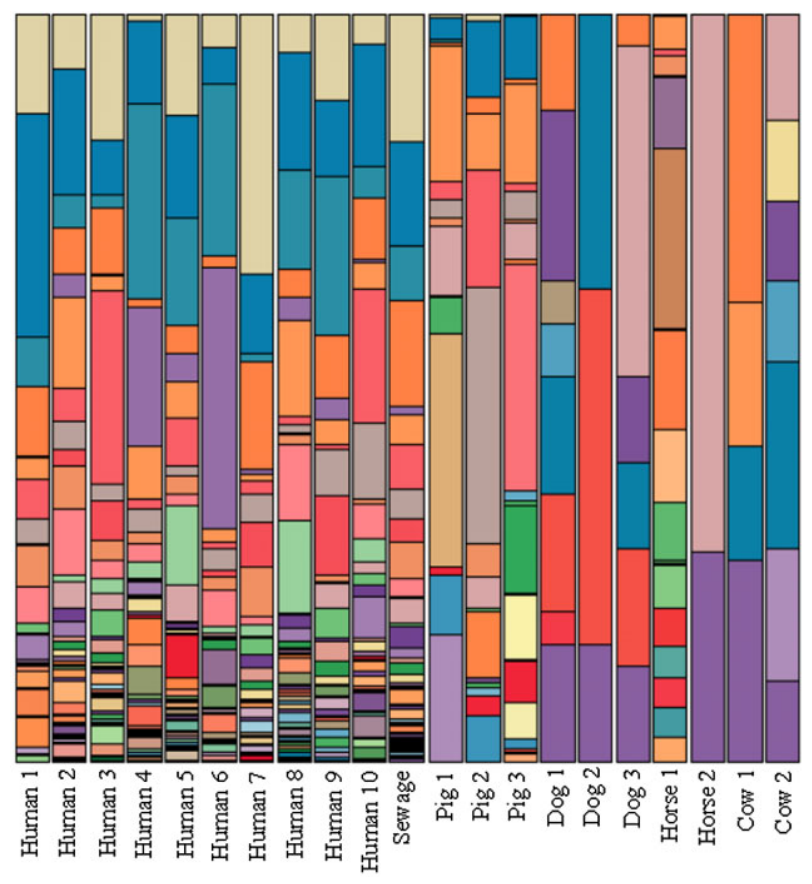

Fig. 2. Distribution of Blautia oligotypes for samples from Brazil sewage and human and animal fecal microbial communities. Oligotyping analysis (Eren et al., 2013b) was performed on 175985 reads identified as Blautia from 27 samples using 15 nucleotide positions following the initial entropy analysis. Base locations of interest based on entropy values included: $1,2,14,15,16,23,24$, $25,31,35,36,38,40,53$ and 56 . To reduce the noise, each oligotype was required to have a most abundant unique sequence with a minimum abundance of 100 . Oligotypes that did not meet this criterion were removed from the analysis. The final number of quality-controlled oligotypes was 81, and they represented 169295 reads (equivalent to $96.2 \%$ of all reads analysed). 


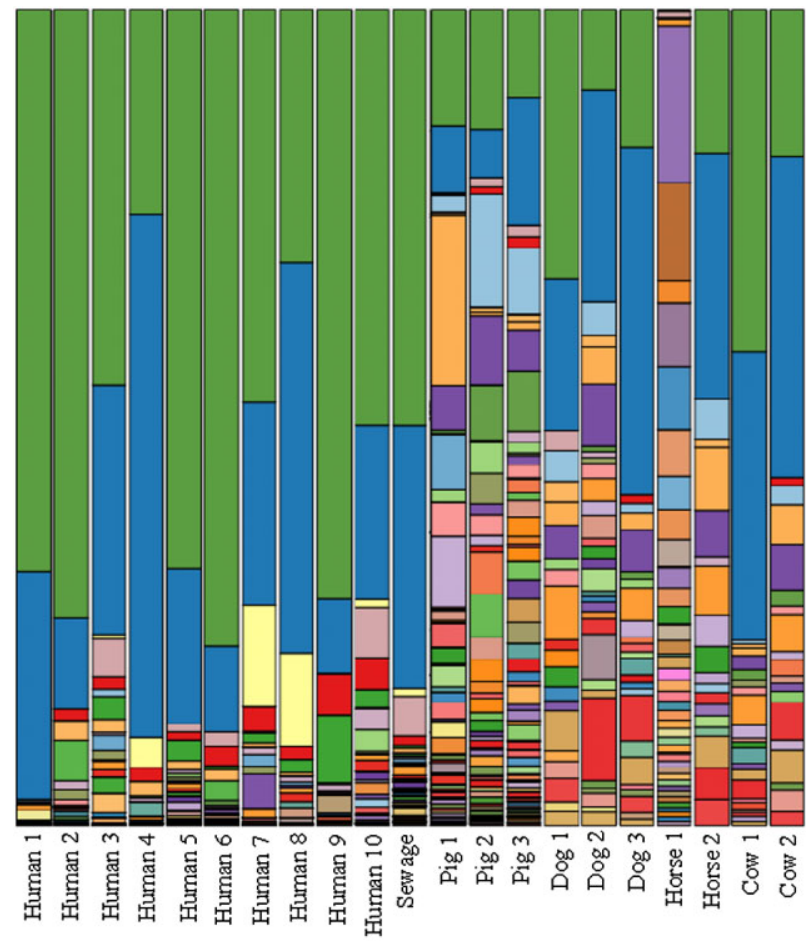

Fig. 3. Distribution of Prevotella oligotypes for samples from Brazil sewage and human and animal fecal microbial communities. Oligotyping analysis was performed on 4676785 reads identified as Prevotella from 27 samples using 19 nucleotide positions following the initial entropy analysis. Base locations of interest included: 10, 13, 15, 17, 19, 20, 21, 23, 24, 25, 27, 34, 35, 37, 38, $40,50,54$ and 55 . To reduce the noise, each oligotype was required to have a most abundant unique sequence with a minimum abundance of 400 . Oligotypes that did not meet this criterion were removed from the analysis. The final number of quality-controlled oligotypes was 188 , and they represented 4641000 reads (equivalent to $99.2 \%$ of all Prevotella reads analysed).

5 and steadily increased at subsequent sites downstream (sites 6 and 7), suggesting cumulative impacts of sewage input. In contrast to the increasing trend of our fecal signature organisms, traditional fecal indicator bacteria populations followed a steady or even decreasing trend. E. coli steadily decreased from sites 3 to 5 , with only a slight increase at sites 6 and 7; and Enterococcus spp. steadily decreased at each downstream site. For this analysis, all sequence reads assigned to the genus Enterococcus were used since $98.5 \%$ of them were not assigned a species. Previous work in our lab has demonstrated that the V6 region may not resolve different species within this genus as $>98 \%$ of presumptive enterococci isolates on $\mathrm{mEl}$ agar were assigned to the genus Enterococcus, but with no species assigned (Koskey et al., 2014).

The human Blautia and Prevotella oligotypes also showed great facility in tracking the human fecal contamination along the Jiquiriçá River. Human-specific/ preferred Blautia and Prevotella oligotypes were low or absent in sites 3 and 4, but appeared at sites 5 to 7 (Fig. 4), consistent with the overall increase in the relative abundance of these two genera.

We also examined the presence of human-specific Blautia and Prevotella oligotypes in the Lucaia River, a historic river in Salvador, Brazil, near the Embasa treatment facility, that now generally appears to be a sewer ditch. This sample had a similar oligotype composition to the Embasa sewage and Jiquiriçá River sites 5 to 7 , demonstrating the prevalence and traceability of Blautia and Prevotella oligotypes in both rural and urban environmental samples. Our cluster analyses illustrated that human feces, Embasa sewage, downstream Jiquiriçá River and Lucaia River samples are similar in Blautia oligotype composition, while the animal fecal (three dogs, three pigs, two cows and one horse) and upstream Jiquiriçá River samples (sites 3 and 4) are similar (Supporting Information Fig. S1). The Prevotella oligotypes also defined distinctions between the human, sewage (Embasa and the Lucaia River) and downstream Jiquiriçá River sites 5 to 7 and the animal samples and upstream Jiquiriçá River sites; however, the similarities were less pronounced than those of the Blautia oligotypes (Supporting Information Fig. S2). These findings further confirm the distinction of human/sewage Blautia oligotypes from animals and demonstrate our ability to track human fecal contamination in the environment.

\section{Conclusions}

We identified Prevotella as the dominant genus in Brazilian human fecal samples and second most abundant in Brazilian sewage. The genus Blautia was also consistently present in human and sewage samples. While both genera have demonstrated their applicability in distinguishing human and animal fecal pollution in Brazil surface waters, Blautia appears to contain more discriminatory and globally applicable markers for human sources. Blautia and Prevotella oligotypes may be a useful combination for identifying source of fecal pollution: Prevotella might function as a 'general' indicator to assess total fecal pollution, while the host-specific/preferred sequences identified in Blautia and Prevotella would assess the amount of human influence. This is particularly important in rural regions of the world, where sewage handling and animal waste are both likely to impact waterways (Esrey et al., 2001). Overall, the notable differences in the taxonomic composition between Brazilian and US human fecal and sewage microbial communities reiterate the need to develop alternative indicators with global applicability for tracking of human fecal pollution in surface waters. 


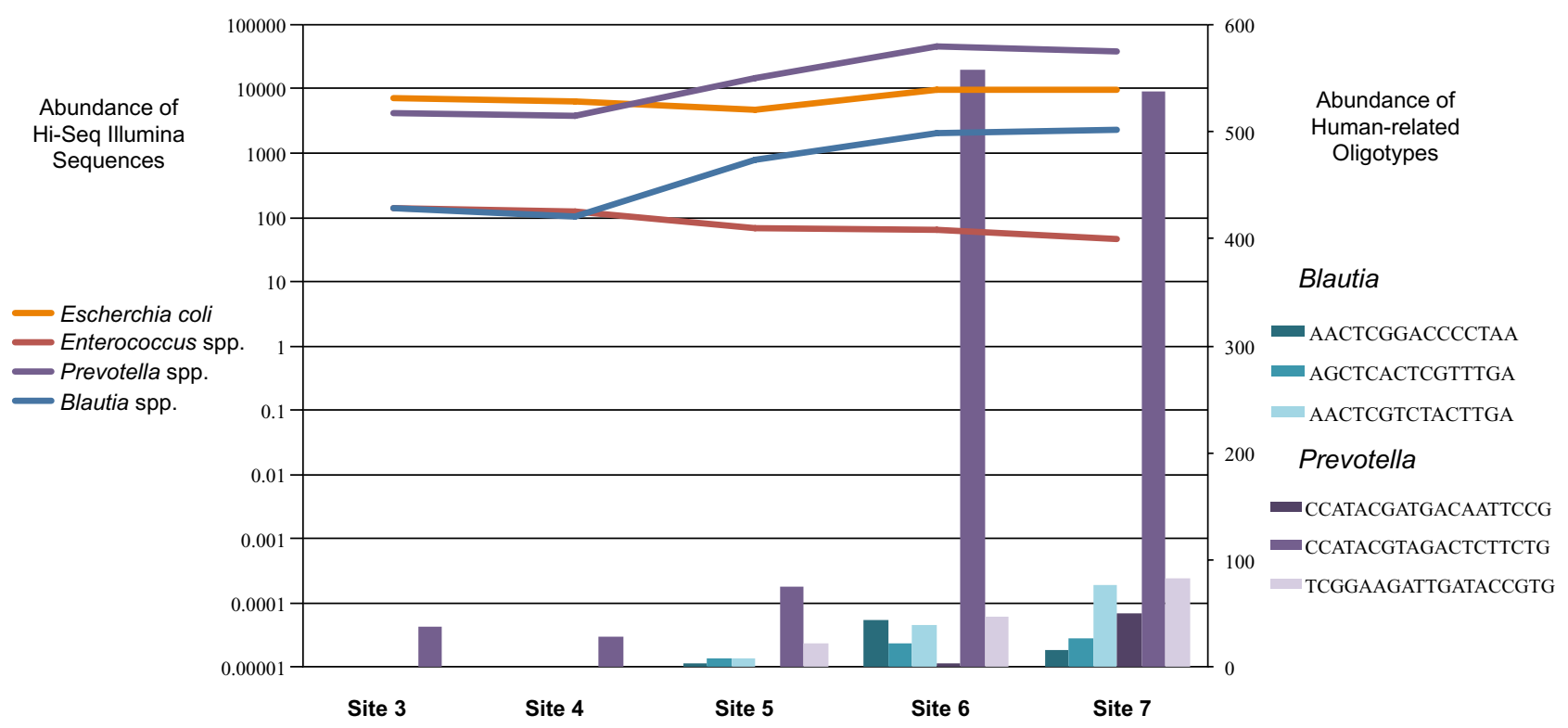

Fig. 4. Human-specific Blautia and Prevotella oligotypes and HiSeq Illumina sequence reads identified as Prevotella spp., Blautia spp. and fecal indicator bacteria (E. coli and Enterococcus spp.) at five sites along the Jiquiriçá River in Jenipapo, Brazil. Blautia oligotypes are shown in shades of blue; Prevotella oligotypes are shown in shades of purple. The water samples were collected on 18 August 2012 as part of a microbial source tracking and schistosomiasis survey (Ponce Terashima et al., unpublished). As with the sewage and fecal samples, sequence reads were generated using HiSeq Illumina sequencing, targeting the V6 hypervariable region of the 16S rRNA gene. Taxonomy was assigned to sequences using GAST, and taxonomic counts were normalized to the maximum number of sequences. Abundance parameters were set from $0 \%$ to $100 \%$; sequence reads are reported on a log-scale. All classified and unclassified (species_NA) species from Prevotella, Blautia and Enterococcus were counted. Sequences classified as E. coli and unclassifiable Enterobacteriaceae (non-distinguishable between Escherichia and Shigella) were added to E. coli counts. The top three human-specific Blautia and human-preferred Prevotella oligotypes are reported as sequence read counts.

\section{Acknowledgements}

This work was supported by the National Oceanic and Atmospheric Administration, Great Lakes Oceans and Human Health Graduate Traineeship Grant to A.M.K. NA06OAR4310119 and through NIH funding on grant R01Al091829-01A1 to S.L.M. We also acknowledge the contributions of Hilary G. Morrison, Sharon L. Grim, Joseph H. Vineis and Mitchell L. Sogin at the Marine Biological Laboratory for sequencing and bioinformatics support.

\section{References}

Ahmed, W., Goonetilleke, A., Powell, D., and Gardner, T. (2009) Evaluation of multiple sewage-associated Bacteroides PCR markers for sewage pollution tracking. Water Res 43: 4872-4877.

Ahmed, W., Yusuf, R., Hasan, I., Goonetilleke, A., and Gardner, T. (2010) Quantitative PCR assay of sewageassociated Bacteroides markers to assess sewage pollution in an urban lake in Dhaka, Bangladesh. Can $J$ Microbiol 56: 838-845.

Barreto, M.L., Genser, B., Strina, A., Teixeira, M.G., Assis, A.M.O., Rego, R.F., et al. (2007) Effect of city-wide sanitation programme on reduction in rate of childhood diarrhoea in northeast Brazil: assessment by two cohort studies. Lancet 370: 1622-1628.

Bernhard, A.E., and Field, K.G. (2000) A PCR assay to discriminate human and ruminant feces on the basis of host differences in Bacteroides-Prevotella genes encoding 16S rRNA. Appl Environ Microbiol 66: 45714574.

Blanton, R.E., Blank, W.A., Costa, J.M., Carmo, T.M., Reis, E.A., Silva, L.K., et al. (2011) Schistosoma mansoni population structure and persistence after praziquantel treatment in two villages of Bahia, Brazil. Int $J$ Parasitol 41: 1093-1099.

De Filippo, C., Cavalieri, D., Di Paola, M., Ramazzotti, M., Poullet, J.B., Massart, S., et al. (2010) Impact of diet in shaping gut microbiota revealed by a comparative study in children from Europe and rural Africa. Proc Natl Acad Sci USA 107: 14691-14696.

Dethlefsen, L., Huse, S., Sogin, M.L., and Relman, D.A. (2008) The pervasive effects of an antibiotic on the human gut microbiota, as revealed by deep $16 \mathrm{~S}$ rRNA sequencing. PLoS Biol 6: e280.

Dick, L.K., Bernhard, A.E., Brodeur, T.J., Santo Domingo, J.W., Simpson, J.M., Walters, S.P., and Field, K.G. (2005) Host distributions of uncultivated fecal Bacteroidales bacteria reveal genetic markers for fecal source identification. Appl Environ Microbiol 71: 3184-3191.

Dubinsky, E.A., Esmaili, L., Hulls, J.R., Cao, Y., Griffith, J.F., and Andersen, G.L. (2012) Application of phylogenetic microarray analysis to discriminate sources of fecal pollution. Environ Sci Technol 46: 4340-4347.

Eren, A.M., Zozaya, M., Taylor, C.M., Dowd, S.E., Martin, D.H., and Ferris, M.J. (2011) Exploring the diversity of Gardnerella vaginalis in the genitourinary tract microbiota 
of monogamous couples through subtle nucleotide variation. PLOS ONE 6: e26732.

Eren, A.M., Vineis, J.H., Morrison, H.G., and Sogin, M.L. (2013a) A filtering method to generate high quality short reads using illumina paired-end technology. PLOS ONE 8: e66643.

Eren, A.M., Maignien, L., Sul, W.J., Murphy, L.G., Grim, S.L., Morrison, H.G., and Sogin, M.L. (2013b) Oligotyping: differentiating between closely related microbial taxa using 16S rRNA gene data. Methods Ecol Evol 4: 11111119.

Eren, A.M., Sogin, M.L., Morrison, H.G., Vineis, J.H., Fisher, J.C., Newton, R.J., and McLellan, S.L. (2014) A single genus in the gut microbiome reflects host preference and specificity. Isme $J$ doi: 10.1038/ismej.2014.97.

Esrey, S.A., Andersson, I., Hillers, A., and Sawyer, R. (2001) Closing the Loop: Ecological Sanitation for Food Security. Publication on Water Resources No. 18, Mexico, Swedish International Development Cooperation Agency.

Field, K.G., and Samadpour, M. (2007) Fecal source tracking, the indicator paradigm, and managing water quality. Water Res 41: 3517-3538.

Fogarty, L.R., and Voytek, M.A. (2005) Comparison of bacteroides-prevotella 16S rRNA genetic markers for fecal samples from different animal species. Appl Environ Microbiol 71: 5999-6007.

Franzolin, M.R., Alves, R.C.B., Keller, R., Gomes, T.A.T., Beutin, L., Barreto, M.L., et al. (2005) Prevalence of diarrheagenic Escherichia coli in children with diarrhea in Salvador, Bahia, Brazil. Mem Inst Oswaldo Cruz 100: 359363.

Fremaux, B., Gritzfeld, J., Boa, T., and Yost, C.K. (2009) Evaluation of host-specific Bacteroidales 16S rRNA gene markers as a complementary tool for detecting fecal pollution in a prairie watershed. Water Res 43: 4838-4849.

Gourmelon, M., Caprais, M.P., Segura, R., Le Mennec, C., Lozach, S., Piriou, J.Y., and Rince, A. (2007) Evaluation of two library-independent microbial source tracking methods to identify sources of fecal contamination in French estuaries. Appl Environ Microbiol 73: 4857-4866.

Guerrant, R.L., Kirchhoff, L.V., Shields, D.S., Nations, M.K., Leslie, J., de Sousa, M.A., et al. (1983) Prospective study of diarrheal illnesses in northeastern Brazil: patterns of disease, nutritional impact, etiologies, and risk factors. $J$ Infect Dis 148: 986-997.

Harwood, V.J., Brownell, M., Wang, S., Lepo, J., Ellender, R.D., Ajidahun, A., et al. (2009) Validation and field testing of library-independent microbial source tracking methods in the Gulf of Mexico. Water Res 43: 4812-4819.

Haugland, R.A., Varma, M., Sivaganesan, M., Kelty, C., Peed, L., and Shanks, O.C. (2010) Evaluation of genetic markers from the 16S rRNA gene $V 2$ region for use in quantitative detection of selected Bacteroidales species and human fecal waste by qPCR. Syst Appl Microbiol 33: 348-357.

Huse, S.M., Dethlefsen, L., Huber, J.A., Welch, D.M., Relman, D.A., and Sogin, M.L. (2008) Exploring microbial diversity and taxonomy using SSU rRNA hypervariable tag sequencing. PLoS Genet 4: e1000255.

Jenkins, M.W., Tiwari, S., Lorente, M., Gichaba, C.M., and Wuertz, S. (2009) Identifying human and livestock sources of fecal contamination in Kenya with host-specific Bacteroidales assays. Water Res 43: 4956-4966.

Kildare, B.J., Leutenegger, C.M., McSwain, B.S., Bambic, D.G., Rajal, V.B., and Wuertz, S. (2007) 16S rRNA-based assays for quantitative detection of universal, human-, cow-, and dog-specific fecal Bacteroidales: a Bayesian approach. Water Res 41: 3701-3715.

Koskey, A.M., Fisher, J.C., Traudt, M.F., Newton, R.J., and McLellan, S.L. (2014) Analysis of the gull fecal microbial community reveals the dominance of Catellicoccus marimammalium in relation to culturable Enterococci. Appl Environ Microbiol 80: 757-765.

Lozupone, C.A., Stombaugh, J.I., Gordon, J.I., Jansson, J.K., and Knight, R. (2012) Diversity, stability and resilience of the human gut microbiota. Nature 489: 220-230.

McLellan, S.L., Huse, S.M., Mueller-Spitz, S.R., Andreishcheva, E.N., and Sogin, M.L. (2010) Diversity and population structure of sewage-derived microorganisms in wastewater treatment plant influent. Environ Microbiol 12: 378-392.

McLellan, S.L., Newton, R.J., Vandewalle, J.L., Shanks, O.C., Huse, S.M., Eren, A.M., and Sogin, M.L. (2013) Sewage reflects the distribution of human faecal Lachnospiraceae. Environ Microbiol 15: 2213-2227.

Mieszkin, S., Yala, J.F., Joubrel, R., and Gourmelon, M. (2010) Phylogenetic analysis of Bacteroidales 16S rRNA gene sequences from human and animal effluents and assessment of ruminant faecal pollution by real-time PCR. J Appl Microbiol 108: 974-984.

Moreno, A.C., Filho, A.F., Gomes Tdo, A., Ramos, S.T., Montemor, L.P., Tavares, V.C., et al. (2010) Etiology of childhood diarrhea in the northeast of Brazil: significant emergent diarrheal pathogens. Diagn Microbiol Infect Dis 66: $50-57$.

Newton, R.J., Vandewalle, J.L., Borchardt, M.A., Gorelick, M.H., and McLellan, S.L. (2011) Lachnospiraceae and Bacteroidales alternative fecal indicators reveal chronic human sewage contamination in an urban harbor. Appl Environ Microbiol 77: 6972-6981.

Newton, R.J., Bootsma, M.J., Morrison, H.G., Sogin, M.L., and McLellan, S.L. (2013) A microbial signature approach to identify fecal pollution in the waters off an urbanized coast of Lake Michigan. Microb Ecol 65: 1011-1023.

de Oliveira, L.K., Fleck, J.D., Comerlato, J., Kluge, M., Bergamaschi, B., Fabres, R.B., et al. (2012) Enteric viruses in water samples from Brazilian dairy farms. Agricultural Water Management 111: 34-39.

Reischer, G.H., Kasper, D.C., Steinborn, R., Mach, R.L., and Farnleitner, A.H. (2006) Quantitative PCR method for sensitive detection of ruminant fecal pollution in freshwater and evaluation of this method in alpine karstic regions. Appl Environ Microbiol 72: 5610-5614.

Reischer, G.H., Kasper, D.C., Steinborn, R., Farnleitner, A.H., and Mach, R.L. (2007) A quantitative real-time PCR assay for the highly sensitive and specific detection of human faecal influence in spring water from a large alpine catchment area. Lett Appl Microbiol 44: 351-356.

Reischer, G.H., Ebdon, J.E., Bauer, J.M., Schuster, N., Ahmed, W., Astrom, J., et al. (2013) Performance characteristics of qPCR assays targeting human- and ruminantassociated bacteroidetes for microbial source tracking 
across sixteen countries on six continents. Environ Sci Technol 47: 8548-8556.

Roslev, P., and Bukh, A.S. (2011) State of the art molecular markers for fecal pollution source tracking in water. Appl Microbiol Biotechnol 89: 1341-1355.

Santo Domingo, J.W., Bambic, D.G., Edge, T.A., and Wuertz, S. (2007) Quo vadis source tracking? Towards a strategic framework for environmental monitoring of fecal pollution. Water Res 41: 3539-3552.

Sauer, E.P., VandeWalle, J.L., Bootsma, M.J., and McLellan, S.L. (2011) Detection of the human specific Bacteroides genetic marker provides evidence of widespread sewage contamination of stormwater in the urban environment. Water Res 45: 4081-4091.

Shanks, O.C., Newton, R.J., Kelty, C.A., Huse, S.M., Sogin, M.L., and McLellan, S.L. (2013) Comparison of the microbial community structures of untreated wastewaters from different geographic locales. Appl Environ Microbiol 79: 2906-2913.

Soller, J.A., Schoen, M.E., Bartrand, T., Ravenscroft, J.E., and Ashbolt, N.J. (2010) Estimated human health risks from exposure to recreational waters impacted by human and non-human sources of faecal contamination. Water Res 44: 4674-4691.

Stewart, J.R., Boehm, A.B., Dubinsky, E.A., Fong, T.T., Goodwin, K.D., Griffith, J.F., et al. (2013) Recommendations following a multi-laboratory comparison of microbial source tracking methods. Water Res 47: 6829-6838.

Turnbaugh, P.J., Hamady, M., Yatsunenko, T., Cantarel, B.L., Duncan, A., Ley, R.E., et al. (2009) A core gut microbiome in obese and lean twins. Nature 457: 480-484.

Unno, T., Jang, J., Han, D., Kim, J.H., Sadowsky, M.J., Kim, O.S., et al. (2010) Use of barcoded pyrosequencing and shared OTUs to determine sources of fecal bacteria in watersheds. Environmental Science \& Technology 44: 7777-7782.

USEPA (2005) Microbial Source Tracking Guide Document. Washington, DC, USA: Office of Research and Development.

VandeWalle, J.L., Goetz, G.W., Huse, S.M., Morrison, H.G., Sogin, M.L., Hoffmann, R.G., et al. (2012) Acinetobacter,
Aeromonas and Trichococcus populations dominate the microbial community within urban sewer infrastructure. Environ Microbiol 14: 2538-2552.

WHO (2007) Combating Waterborne Disease at the Household Level. Geneva, Switzerland: World Health Organization.

WHO (2013a) Schistosomiasis Fact Sheet. Geneva, Switzerland: World Health Organization. [WWW document]. URL: http://www.who.int/mediacentre/factsheets/fs115/en/ index.html)

WHO (2013b) Progress on Sanitation and DrinkingWater 2013 Update. Geneva, Switzerland: World Health Organization.

Wu, G.D., Chen, J., Hoffmann, C., Bittinger, K., Chen, Y.-Y., Keilbaugh, S.A., et al. (2011) Linking long-term dietary patterns with gut microbial enterotypes. Science 334: 105108.

Yatsunenko, T., Rey, F.E., Manary, M.J., Trehan, I., Dominguez-Bello, M.G., Contreras, M., et al. (2012) Human gut microbiome viewed across age and geography. Nature 486: 222-227.

\section{Supporting information}

Additional Supporting Information may be found in the online version of this article at the publisher's web-site:

Fig. S1. Hierarchical cluster analysis of 27 samples (10 humans, 10 animals, one sewage (Embasa), one sewage outfall and five river samples) with respect to Blautia oligotypes they possess. Distances between samples were determined using Canberra distance metric. The fiver river oligotypes cluster more closely with animals upstream and with humans downstream after passing through the village.

Fig. S2. Hierarchical cluster analysis of 27 samples (10 humans, 10 animals, one sewage (Embasa), one sewage outfall and five river samples) with respect to Prevotella oligotypes they possess. Distances between samples were determined using Canberra distance metric.

Text S1. Sample collection processing and DNA extraction. 